\title{
Inferring an optimal Fisher measure
}

\author{
S.P. Flego ${ }^{2}$, A. Plastino ${ }^{1,3,5}$, and A. R. Plastino ${ }^{3,4}$ \\ 1 Universidad Nacional de La Plata, \\ Instituto de Física (IFLP-CCT-CONICET), \\ C.C. 727, 1900 La Plata, Argentina \\ 2 Universidad Nacional de La Plata, \\ Fac. de Ingeniería, 1900 La Plata, Argentina \\ 3 CREG-University of La Plata-CONICET \\ C.C. 727, 1900 La Plata, Argentina \\ 4 Instituto Carlos I de Fisica Teorica y Computacional and \\ Departamento de Fisica Atomica, Molecular y Nuclear, \\ Universidad de Granada, Granada, Spain \\ 5 Universitat de les Illes Balears and IFISC-CSIC, 07122 Palma de Mallorca, Spain
}

\begin{abstract}
It is well known that a suggestive relation exists that links Schrödinger's equation (SE) to the information-optimizing principle based on Fisher's information measure (FIM). We explore here an approach that will allow one to infer the optimal FIM compatible with a given amount of prior information without explicitly solving first the associated SE. This technique is based on the virial theorem and it provides analytic solutions for the physically relevant FIM, that which is minimal subject to the constraints posed by the prior information.
\end{abstract}

KEYWORDS: Information Theory, Fisher's Information measure, Legendre transform, Virial theorem. 


\section{INTRODUCTION}

Although Fisher's information measure (FIM) I dates from the 20's, it has attracted intense attention from physicists only since the mid-90's [1-3]. Interest in FIM's physical applications [3 9] has been growing exponentially since the appearance of Frieden and Soffer's seminal paper [5]. A very small, and certainly not exhaustive sample is that of Refs. [10 24]. The mathematical problem of extremizing $I$ under given constraints has proved to be relevant in connection with several scenarios related to quantum mechanics and statistical physics, as the above cited references indicate. We intend to exhibit novel links between $I$ and the Schrödinger equation that, via the virial theorem, lead in natural fashion to a differential equation for $I$. Such equation, that has an analytical solution, encodes the available prior knowledge concerning the system at hand in terms of adequately selected expectation values. Our solution adds to the rather large Fisher literature a general, explicit expression for that particular FIM $I_{M i n}$ that arises out of any constrained $I$-extremization problem.

To better understand why this is of importance one should recall that Fisher's information and Shannon's entropy play complementary roles [4]. The former is convex, the later concave. When one grows, the other diminishes, etc. The associated Shannon's MaxEnt problem has as its solution, always, an exponential form that contains those physical quantities whose mean values are a priori known. The solution of the FIM minimization problem is instead a Schrödinger like differential equation [6, 7, 9], whose solutions exhibit a panoply of different mathematical forms. We provide here special definite forms for $I_{M i n}$, expressed in terms of those mean values that are a priori known, filling thus a gap in the literature of the physics of information. We review basic materials below and start with our presentation in Section III-IV. We give illustrative examples in Section $\mathrm{V}$ and discuss particular issues in Section VI. Our conclusions are given in Section VII.

\section{REVIEW OF BASIC IDEAS}

We briefly review here the formalism developed in Ref. [6] (see also [7]). Consider a system that is specified by a physical parameter $\theta$ and let $f(x, \theta)$ describe the normalized probability distribution function (PDF) for this parameter. If an observer were to make a measurement of $x$ and had to best infer $\theta$ from such measurement, calling the resulting estimate $\tilde{\theta}=\tilde{\theta}(x)$, 
one might well wonder how well $\theta$ could be determined. Estimation theory [4] asserts that the best possible estimator $\tilde{\theta}(x)$, after a very large number of $x$-samples is examined, suffers a mean-square error $e^{2}$ from $\theta$ obeying the rule $I e^{2}=1$, where the Fisher information measure (FIM) $I$, a functional of the PDF, reads

$$
I=\int d x f(x, \theta)\left\{\frac{\partial}{\partial \theta} \ln [f(x, \theta)]\right\}^{2} .
$$

Any other estimator must have a larger mean-square error (all estimators must be unbiased, i.e., satisfy $\langle\tilde{\theta}(\mathbf{x})\rangle=\theta)$. Thus, FIM has a lower bound. No matter what the parameter $\xi$ of the system might be, $I$ has to obey

$$
I e_{\xi}^{2} \geq 1
$$

the celebrated Cramer-Rao bound [4]. The particular instance of translational families merits a word. They are mono-parametric distribution families of the form $f(x, \theta)=f(x-\theta)$, known up to the shift parameter $\theta$. All family members exhibit identical shape. After introducing the amplitudes $\psi$ such that $f(x)=\psi(x)^{2}$, FIM adopts the simpler aspect [5]

$$
I=\int d x f(x)\left\{\frac{\partial}{\partial x} \ln [f(x)]\right\}^{2}=4 \int d x\left[\psi^{\prime}(x)\right]^{2} ; \quad\left(d \psi / d x=\psi^{\prime}\right) .
$$

Note that for the uniform distribution $f(x)=$ constant one has $I=0$. Focus attention now a system that is specified by a set of $M$ physical parameters $\mu_{k}$. We can write $\mu_{k}=\left\langle A_{k}\right\rangle$ with $A_{k}=A_{k}(x)$. The set of $\mu_{k}$-values constitutes the prior knowledge. It represents available empirical information. Let the pertinent probability distribution function (PDF) be $f(x)$. Then,

$$
\left\langle A_{k}\right\rangle=\int d x A_{k}(x) f(x), \quad k=1, \ldots, M .
$$

In this context it can be shown (see for example [6, 9]) that the physically relevant PDF $f(x)$ minimizes the FIM (3) subject to the prior conditions and the normalization condition. In the celebrated MaxEnt approach of Jaynes' [25] one maximizes the entropy, that behaves information-wise in opposite fashion to that of Fisher's measure [5]. Normalization entails $\int d x f(x)=1$, and, consequently, our Fisher-based extremization problem adopts the appearance

$$
\delta\left(I-\alpha \int d x f(x)-\sum_{k=1}^{M} \lambda_{k} \int d x A_{k}(x) f(x)\right)=0
$$

where we have introduced the $(M+1)$ Lagrange multipliers $\lambda_{k}\left(\lambda_{0}=\alpha\right)$. In Ref. [6] on can find the details that lead from (5) to a Schrödinger's equation (SE) that yields the desired PDF in terms of an amplitude $\psi(x)$ referred to above before Eq. (3). This SE is of the form 


$$
-\frac{1}{2} \frac{\partial^{2}}{\partial x^{2}} \psi-\sum_{k=1}^{M} \frac{\lambda_{k}}{8} A_{k} \psi=\frac{\alpha}{8} \psi
$$

which can be formally interpreted as the (real) Schrödinger equation for a particle of unit mass $(\hbar=1)$ moving in the effective, "information-related pseudo-potential" [6]

$$
U=U(x)=-\frac{1}{8} \sum_{k=1}^{M} \lambda_{k} A_{k}(x)
$$

in which the normalization-Lagrange multiplier $(\alpha / 8)$ plays the role of an energy eigenvalue. The $\lambda_{k}$ are fixed, of course, by recourse to the available prior information. Note that $\psi(x)$ is always real in the case of one-dimensional scenarios, or for the ground state of a real potential in $\mathrm{N}$ dimensions [26]. In terms of the amplitudes $\psi(x)$ we have

$$
\begin{aligned}
I & =\int d x f\left(\frac{\partial \ln f}{\partial x}\right)^{2}=\int d x \psi_{n}^{2}\left(\frac{\partial \ln \psi_{n}^{2}}{\partial x}\right)^{2}=4 \int d x\left(\frac{\partial \psi_{n}}{\partial x}\right)^{2}= \\
& =-4 \int \psi_{n} \frac{\partial^{2}}{\partial x^{2}} \psi_{n} d x=-4\left\langle\frac{\partial^{2}}{\partial x^{2}}\right\rangle=\int \psi_{n}\left(\alpha+\sum_{k=1}^{M} \lambda_{k} A_{k}\right) \psi_{n} d x
\end{aligned}
$$

i.e.,

$$
I=\alpha+\sum_{k=1}^{M} \lambda_{k}\left\langle A_{k}\right\rangle
$$

a form that we will employ in our developments below. The connection between our variational solutions $f$ and thermodynamics was established in Refs. [6] and [8] in the guise of reciprocity relations that express the Legendre-transform structure of thermodynamics. They constitute its essential formal ingredient [27] and were re-derived à la Fisher in [6] by recasting (8) in a fashion that emphasizes the relevant independent variables

$$
I\left(\left\langle A_{1}\right\rangle, \ldots,\left\langle A_{M}\right\rangle\right)=\alpha+\sum_{k=1}^{M} \lambda_{k}\left\langle A_{k}\right\rangle .
$$

The Legendre transform changes the relevant variables. In the case of $I$ this is

$$
\alpha=I\left(\left\langle A_{1}\right\rangle, \ldots,\left\langle A_{M}\right\rangle\right)-\sum_{k=1}^{M} \lambda_{k}\left\langle A_{k}\right\rangle=\alpha\left(\lambda_{1}, \ldots, \lambda_{M}\right),
$$

so that we encounter the three reciprocity relations proved in [6] 


$$
\frac{\partial \alpha}{\partial \lambda_{i}}=-\left\langle A_{i}\right\rangle ; \quad \lambda_{k}=\frac{\partial I}{\partial\left\langle A_{k}\right\rangle} ; \quad \frac{\partial I}{\partial \lambda_{i}}=\sum_{k}^{M} \lambda_{k} \frac{\partial\left\langle A_{k}\right\rangle}{\partial \lambda_{i}},
$$

the last one being a generalized Fisher-Euler theorem.

\section{PRESENT CORE-RESULTS}

Our previous Fisher considerations lead to a scenario for which

$$
H=-\frac{1}{2} \frac{\partial^{2}}{\partial x^{2}}+U(x), \quad U(x)=-\frac{1}{8} \sum_{k} \lambda_{k} A_{k}, \quad E_{n}=\frac{\alpha}{8}
$$

Enters here, as essential new ingredient in the present considerations, the celebrated virial theorem [28] that of course applies in this Schrödinger-scenario [29]. This theorem is intimately related to the reciprocity relations of the preceding Section, as discussed in [30] and states that

$$
\left\langle-\frac{\partial^{2}}{\partial x^{2}}\right\rangle=\left\langle x \frac{\partial}{\partial x} U(x)\right\rangle .
$$

The potential function $U(x)$ belongs to $\mathcal{L}_{2}$ and thus admit of a series expansion in $x, x^{2}, x^{3}$, etc. [29]. The $A_{k}(x)$ themselves belong to $\mathcal{L}_{2}$ as well and can be series-expanded in similar fashion. This enables us to base our future considerations on the assumption that the a priori knowledge refers to moments $x^{k}$ of the independent variable, i.e.,

$$
\left\langle A_{k}\right\rangle=\left\langle x^{k}\right\rangle
$$

and that one possesses information on $M$ moment-mean values $\left\langle x^{k}\right\rangle$. Our "information" potential $U$ then reads

$$
U(x)=-\frac{1}{8} \sum_{k} \lambda_{k} x^{k}
$$

and Eq. (13) allows one to immediately obtain

$$
\left\langle\frac{\partial^{2}}{\partial x^{2}}\right\rangle=\frac{1}{8} \sum_{k=1}^{M} k \lambda_{k}\left\langle A_{k}\right\rangle ; \quad\left(A_{k}=x^{k}\right)
$$

and thus, via (16) and the above mentioned relation $I=-4\left\langle\partial_{x x}\right\rangle$, a useful, virial-related expression for Fisher's information measure can be arrived at. 


$$
I=-\sum_{k=1}^{M} \frac{k}{2} \lambda_{k}\left\langle x^{k}\right\rangle
$$

which is an explicit function of the M physical parameters $\left\langle x^{k}\right\rangle$ and their respective Lagrange multipliers $\lambda_{k}$. Eq. (17) encodes the information provided by the Virial theorem. Thus, we have two different ways of expressing $I$, namely, (8) and (17). Interesting things happen if we put them together. Since $\lambda_{k}$ is given by (11) as $\left[\partial I / \partial\left\langle x^{k}\right\rangle\right]$, inserting the reciprocity relations (11) into (17) we are led to

$$
\frac{\partial I}{\partial\left\langle x^{k}\right\rangle}=\lambda_{k} \quad \longrightarrow \quad I=-\sum_{k=1}^{M} \frac{k}{2}\left\langle x^{k}\right\rangle \frac{\partial I}{\partial\left\langle x^{k}\right\rangle}
$$

Eq. (18) constitutes an important result, since we have now at our disposal a differential FIM-equation. Dealing with it should allow us to find $I$ in terms of the $\left\langle x^{k}\right\rangle$ without passing first through a Schrödinger equation first, a commendable achievement. This is a linear partial differential equation that an extremal $I$ must necessarily comply with. This constitutes one of the main present results. It is not clear (yet) whether from such an $I$-form we can extract an amplitude $\psi$ satisfying a Schrödinger equation. Our I could however be related to an approximate solution to Schrödinger's equation. For convenience we now recast our key relations using dimensionless magnitudes

$$
\forall\left\langle A_{k}\right\rangle \equiv\left\langle x^{k}\right\rangle \neq 0, \quad \mathcal{I}=\frac{I}{[I]}=\frac{I}{[x]^{2}} \quad, \quad\left\langle\mathcal{X}_{k}\right\rangle=\frac{\left\langle x^{k}\right\rangle}{\left[\left\langle x^{k}\right\rangle\right]}=\frac{\left\langle x^{k}\right\rangle}{[x]^{k}},
$$

where $[I]$ and $\left[\left\langle x^{k}\right\rangle\right]$ denote the dimension of $I$ and $\left\langle x^{k}\right\rangle$, respectively. Thus, the differential equation that governs the FIM-behavior, i.e., (18), can be translated into

$$
\mathcal{I}=-\sum_{k=1}^{M} \frac{k}{2}\left\langle\mathcal{X}_{k}\right\rangle \frac{\partial \mathcal{I}}{\partial\left\langle\mathcal{X}_{k}\right\rangle}, \quad \mathcal{I}=\mathcal{I}\left(\left\langle\mathcal{X}_{1}\right\rangle, \cdots,\left\langle\mathcal{X}_{M}\right\rangle\right)
$$

which is a first order linear nonhomogeneous equation with $M$ independent variables. All first order, linear partial differential equations (PDEs) possess a solution that depends on an arbitrary function, called the general solution of the PDE. In many physical situations this solution if less important than other solutions called complete ones [32 34]. Such complete solutions are particular PDE solutions containing as many arbitrary constants as intervening independent variables. Let us look now for a special complete solution of our PDE (20), 
whose usefulness will be illustrated via two physical examples below (the treatment of general solutions is postponed to Section VI). We first set

$$
\mathcal{I}=\sum_{k=1}^{M} \mathcal{I}_{k}=\sum_{k=1}^{M} \exp \left[g\left(\left\langle\mathcal{X}_{k}\right\rangle\right)\right]
$$

and substituting (21) into (18) leads to

$$
\mathcal{I}=-\sum_{k=1}^{M} \frac{k}{2}\left\langle\mathcal{X}_{k}\right\rangle g^{\prime}\left(\left\langle\mathcal{X}_{k}\right\rangle\right) \mathcal{I}_{k}
$$

The above relation entails

$$
g^{\prime}\left(\left\langle\mathcal{X}_{k}\right\rangle\right)=-\frac{2}{k\left\langle\mathcal{X}_{k}\right\rangle} \quad \longrightarrow \quad g\left(\left\langle\mathcal{X}_{k}\right\rangle\right)=-\frac{2}{k} \ln \left|\left\langle\mathcal{X}_{k}\right\rangle\right|+c_{k},
$$

where $c_{k}$ is an integration constant. Finally, substituting (23) into (21) we arrive at

$$
\mathcal{I}=\sum_{k=1}^{M} C_{k} \exp \left(-\frac{2}{k} \ln \left|\left\langle\mathcal{X}_{k}\right\rangle\right|\right), \quad C_{k}=e^{c_{k}}>0
$$

which can be recast as

$$
\mathcal{I}\left(\left\langle\mathcal{X}_{1}\right\rangle, \ldots,\left\langle\mathcal{X}_{M}\right\rangle\right)=\sum_{k=1}^{M} C_{k}\left|\left\langle\mathcal{X}_{k}\right\rangle\right|^{-2 / k}
$$

or, in function of the original input-quantities (19)

$$
I\left(\left\langle x^{1}\right\rangle, \ldots,\left\langle x^{M}\right\rangle\right)=\sum_{k=1}^{M} C_{k}\left|\left\langle x^{k}\right\rangle\right|^{-2 / k},
$$

an intriguing result. We enumerate below the main properties of this minimal $I$.

\section{- FIM-domain}

Obviously, it is

$$
\operatorname{Dom}[I]=\left\{\left(\left\langle x^{1}\right\rangle, \ldots,\left\langle x^{M}\right\rangle\right) /\left\langle x^{k}\right\rangle \in \Re_{o}\right\}
$$

\section{- FIM-monotonicity}

Differentiating (26) we obtain

$$
\frac{\partial I}{\partial\left\langle x^{k}\right\rangle}=-\frac{2}{k\left\langle x^{k}\right\rangle} I_{k}=-\frac{2}{k\left\langle x^{k}\right\rangle} C_{k}\left|\left\langle x^{k}\right\rangle\right|^{-2 / k} .
$$


Therefore, if $\left\langle x^{k}\right\rangle>0, I$ is a monotonically decreasing function in the $\left\langle x^{k}\right\rangle$-direction. Also, for $\left\langle x^{k}\right\rangle>0$, from the reciprocity relations (11) we have,

$$
\lambda_{k}=-\frac{2}{k} C_{k}\left\langle x^{k}\right\rangle^{-(2+k) / k}<0 .
$$

\section{- FIM-convexity}

This is a necessary property, since the entropy is concave. By differentiation of the expression (27) one obtains

$$
\frac{\partial^{2} I}{\partial\left\langle x^{n}\right\rangle \partial\left\langle x^{k}\right\rangle}=\left(1+\frac{k}{2}\right) \frac{4}{k^{2}} C_{k}\left|\left\langle x^{k}\right\rangle\right|^{-2(1+k) / k} \delta_{k n},
$$

from which we can assert that the Fisher measure is a convex function. It is then guaranteed that the inverse of $\partial_{k} \partial_{j} \bar{\alpha}$ exists.

\section{THE REFERENCE QUANTITIES $C_{k}$}

FIM is an estimation measure known to obey the Cramer Rao-bound (21) [4]. The best estimator exhibits a CR relation as close to unity as possible. Thus, the reference quantities $C_{k}$ should be chosen in a manner that respects this condition. Here we are interested in simple situations that illustrate the concomitant procedure. More involved situations will be treated elsewhere.

Since the reference quantities $C_{k}$ contain important information concerning the reference system with respect the which prior conditions are experimentally determined, it is convenient to start by choosing an appropriate reference one.

\section{Minimum of the information potential}

We consider it reasonable to incorporate at the outset, within the $I$-form, information concerning the minimum of the information potential $U(x)$. Assume that this informationpotential

$$
U(x)=-\frac{1}{8} \sum_{x=1}^{M} \lambda_{k} x^{k}
$$

achieves its absolute minimum at the "critical point" $x=\xi$,

$$
U^{\prime}(\xi)=0, \quad U_{\min }=U(\xi)
$$


Effecting the FIM-translational transform $u=x-\xi$ leads us to

$$
I=-\sum_{k=1}^{M} \frac{k}{2} \lambda_{k}\left\langle x^{k}\right\rangle=-\sum_{k=1}^{M} \frac{k}{2} \lambda_{k}^{*}\left\langle u^{k}\right\rangle^{\prime}
$$

with (see the Appendix)

$$
\lambda_{k}^{*}=-\frac{8}{k !} U^{(k)}(\xi), \quad\left\langle u^{k}\right\rangle^{\prime}=\left\langle(x-\xi)^{k}\right\rangle
$$

where $U^{(k)}(\xi)$ is the $k^{\text {th }}$ derivative of $\mathrm{U}(\mathrm{x})$ evaluated at $x=\xi$ and \langle\rangle$^{\prime}$ indicates that the pertinent mean value $(x$-moment) is evaluated for translation-transformed eigenfunctions. The corresponding FIM-explicit functional expression is built up with the $N$-non-vanishing momenta $(N<M)\left(\left\langle u^{k}\right\rangle^{\prime} \neq 0\right)$ and is given by

$$
I=\sum_{k=2}^{N} C_{k}\left|\left\langle u^{k}\right\rangle^{\prime}\right|^{-2 / k}=\sum_{k=2}^{N} C_{k}\left|\left\langle(x-\xi)^{k}\right\rangle\right|^{-2 / k}
$$

where we kept in mind that $\lambda_{1}^{*}=-8 U^{\prime}(\xi)=0$. A glance at the above expression suggests that we re-arrange things in the fashion

$$
I=C_{2}\left|\left\langle(x-\xi)^{2}\right\rangle\right|^{-1}+\sum_{k=3}^{N} C_{k}\left|\left\langle(x-\xi)^{k}\right\rangle\right|^{-2 / k}
$$

Taking now into account that

$$
\left\{\begin{array} { l } 
{ \langle x - \xi \rangle = 0 } \\
{ \langle ( x - \xi ) ^ { 2 } \rangle = \langle x ^ { 2 } \rangle - 2 \xi \langle x \rangle + \xi ^ { 2 } }
\end{array} \longrightarrow \quad \left\{\begin{array}{l}
\langle x\rangle=\xi \\
\left\langle(x-\xi)^{2}\right\rangle=\left\langle x^{2}\right\rangle-\langle x\rangle^{2}=\sigma^{2}
\end{array}\right.\right.
$$

we get

$$
I=\frac{C_{2}}{\sigma^{2}}+\sum_{k=3}^{N} C_{k}\left|\left\langle(x-\xi)^{k}\right\rangle\right|^{-2 / k}
$$

from which we obtain

$$
I \sigma^{2}=C_{2}+\sigma^{2} \sum_{k=3}^{N} C_{k}\left|\left\langle(x-\xi)^{k}\right\rangle\right|^{-2 / k} \geq 1 .
$$

Therefore, $I$ preserves the well-known Cramer-Rao $I$-bound [5] $I \sigma^{2} \geq 1$. The above seems to indicate that if no moment $k \geq 3$ is a priori known, the lower bound can be reached for $C_{2}=1$. For $k \geq 3$ additional considerations apply that will be discussed elsewhere. 


\section{TWO PHYSICAL EXAMPLES}

So as to illustrate the above considerations we are going to consider two simple and instructive examples. We take the mass $m=1$ and $\hbar=1$.

\section{Harmonic oscillator (HO)}

The prior information is given by

$$
\left\langle x^{2}\right\rangle=\frac{1}{2 \omega}, \quad M=1, \quad k=2 .
$$

The minimum of the potential function obtains at the origin $\xi=0$,

$$
U(x)=-\frac{1}{8} \lambda_{2} x^{2} \quad \longrightarrow \quad U^{\prime}(\xi)=-\frac{1}{4} \lambda_{2} \xi=0 \quad \longrightarrow \quad \xi=0
$$

The pertinent FIM can be obtained using (33) with $u=x-\xi=x$,

$$
I=I\left(\left\langle x^{2}\right\rangle\right)=C_{2}\left\langle x^{2}\right\rangle^{-1}
$$

and, the CR bound is saturated when $C_{2}=1$,

$$
I\left\langle x^{2}\right\rangle=C_{2}=1 \quad \Longrightarrow \quad I=\left\langle x^{2}\right\rangle^{-1}
$$

The corresponding Lagrange multiplier can be obtained by recourse to the reciprocity relations (11) and (39),

$$
\lambda_{2}=\frac{\partial I}{\partial\left\langle x^{2}\right\rangle}=-\left\langle x^{2}\right\rangle^{-2} .
$$

The prior-knowledge (38) is encoded into the FIM (39), and the Lagrange multiplier $\lambda_{2}$ (40),

$$
I=\left\langle x^{2}\right\rangle^{-1}=2 \omega ; \quad \lambda_{2}=-\left\langle x^{2}\right\rangle^{-2}=-4 \omega^{2}
$$

and the $\alpha$-value can be obtained from (10),

$$
\alpha=I-\lambda_{2}\left\langle x^{2}\right\rangle=4 \omega
$$

as we expect. 


\section{Harmonic oscillator in a uniform field}

We consider a charged unit-mass particle moving in the HO potential. The electrical charge is $\mathrm{q}$ and there is a uniform electric field $\epsilon$, in the $x$-direction. Our prior knowledge is given by [29]

$$
\langle x\rangle=\frac{q \epsilon}{\omega^{2}}, \quad\left\langle x^{2}\right\rangle=\frac{1}{2 \omega}+\left(\frac{q \epsilon}{\omega^{2}}\right)^{2} .
$$

We look first for the $\xi$-point at which $U(x)$ is minimal.

$$
\begin{gathered}
U(x)=-\frac{1}{8}\left(\lambda_{1} x+\lambda_{2} x^{2}\right) \\
U^{\prime}(\xi)=-\frac{1}{8}\left(\lambda_{1}+2 \lambda_{2} \xi\right)=0 \quad \longrightarrow \quad \xi=-\frac{\lambda_{1}}{2 \lambda_{2}} .
\end{gathered}
$$

The translational transform $u=x-\xi$ implies that

$$
\langle u\rangle^{\prime}=\langle x-\xi\rangle=\langle x\rangle-\xi, \quad\left\langle u^{2}\right\rangle^{\prime}=\left\langle(x-\xi)^{2}\right\rangle=\left\langle x^{2}\right\rangle-2 \xi\langle x\rangle+\xi^{2}
$$

The translation transformed FIM is now given by

$$
I=C_{2}\left\langle u^{2}\right\rangle^{\prime}-1
$$

and, the CR bound is saturated when $C_{2}=1$,

$$
I\left\langle u^{2}\right\rangle^{\prime}=C_{2}=1 \quad \Longrightarrow \quad I=\left\langle u^{2}\right\rangle^{\prime-1}
$$

The reciprocity relations lead us to

$$
\begin{aligned}
& \lambda_{1}=\frac{\partial I}{\partial\langle x\rangle}=\frac{\partial I}{\partial\left\langle u^{2}\right\rangle^{\prime}} \frac{\partial\left\langle u^{2}\right\rangle^{\prime}}{\partial\langle x\rangle}=-\left\langle u^{2}\right\rangle^{\prime}-2(-2 \xi) \\
& \lambda_{2}=\frac{\partial I}{\partial\left\langle x^{2}\right\rangle}=\frac{\partial I}{\partial\left\langle u^{2}\right\rangle^{\prime}} \frac{\partial\left\langle u^{2}\right\rangle^{\prime}}{\partial\left\langle x^{2}\right\rangle}=-\left\langle u^{2}\right\rangle^{\prime}-2
\end{aligned}
$$

From the prior knowledge (43) and using (45) we have

$$
\begin{gathered}
\langle x\rangle=\xi=\frac{q \epsilon}{\omega^{2}} \\
\left\langle u^{2}\right\rangle^{\prime}=\left\langle x^{2}\right\rangle-\xi^{2}=\frac{1}{2 \omega}+\left(\frac{q \epsilon}{\omega^{2}}\right)^{2}-\left(\frac{q \epsilon}{\omega^{2}}\right)^{2}=\frac{1}{2 \omega},
\end{gathered}
$$


then, inserting (50) and (51) into (47) - (49) we get

$$
\begin{gathered}
I=\left\langle u^{2}\right\rangle^{\prime-1}=\left(\frac{1}{2 \omega}\right)^{-1}=2 \omega, \\
\lambda_{1}=2 \xi\left\langle u^{2}\right\rangle^{\prime}=2 \frac{q \epsilon}{\omega^{2}}(2 \omega)^{2}=8 q \epsilon \\
\lambda_{2}=-\left\langle u^{2}\right\rangle^{\prime-2}=-(2 \omega)^{2}=-4 \omega^{2}
\end{gathered}
$$

The corresponding translational transform $\bar{\alpha}$-value can be obtained substituting (52)-(54) into (10),

$$
\bar{\alpha}=I-\lambda_{1}\langle x\rangle-\lambda_{2}\left\langle x^{2}\right\rangle=2 \omega-8 q \epsilon \frac{q \epsilon}{\omega^{2}}+4 \omega^{2}\left(\frac{1}{2 \omega}+\left(\frac{q \epsilon}{\omega^{2}}\right)^{2}\right)=4 \omega,
$$

and the corresponding $\alpha$-value is given by (see Appendix),

$$
\alpha=\bar{\alpha}+8 U(\xi)=4 \omega-4 \frac{q^{2} \epsilon^{2}}{\omega^{2}}
$$

as we expect.

\section{GENERAL SOLUTION OF THE DIFFERENTIAL FIM-EQUATION}

We discuss here this issue for the sake of completeness. Our FIM-equation is a first order linear nonhomogeneous differential equation. We are following $\left[32 \_34\right]$ in looking for the general solution. For a first-order PDE, the method of characteristics allows one to encounter useful curves (called characteristic curves or just characteristics) along which the PDE becomes an ordinary differential equation (ODE). Once the ODE is found, it can be solved along the characteristic curves and transformed into a solution for the original PDE. The characteristic system of Eq. (20) is

$$
-\frac{d\left\langle\mathcal{X}_{i}\right\rangle}{(i / 2)\left\langle\mathcal{X}_{i}\right\rangle}=-\frac{d\left\langle\mathcal{X}_{j}\right\rangle}{(j / 2)\left\langle\mathcal{X}_{j}\right\rangle}=\frac{d \mathcal{I}}{\mathcal{I}}, \quad i, j=1, \cdots, M
$$

leads (for $\left\langle\mathcal{X}_{1}\right\rangle \neq 0$ ) to

$$
\begin{aligned}
\frac{d\left\langle\mathcal{X}_{k}\right\rangle}{(k / 2)\left\langle\mathcal{X}_{k}\right\rangle}=\frac{d\left\langle\mathcal{X}_{1}\right\rangle}{(1 / 2)\left\langle\mathcal{X}_{1}\right\rangle} \longrightarrow \frac{2}{k} \ln \left|\left\langle\mathcal{X}_{k}\right\rangle\right|+c_{k} & =2 \ln \left|\left\langle\mathcal{X}_{1}\right\rangle\right|+c_{1} \\
\ln \left[e^{c_{k}}\left|\left\langle\mathcal{X}_{k}\right\rangle\right|^{2 / k}\right] & =\ln \left[e^{c_{1}}\left|\left\langle\mathcal{X}_{1}\right\rangle\right|^{2}\right] \\
e^{c_{k}}\left|\left\langle\mathcal{X}_{k}\right\rangle\right|^{2 / k} & =e^{c_{1}}\left|\left\langle\mathcal{X}_{1}\right\rangle\right|^{2}
\end{aligned}
$$




$$
\begin{aligned}
b_{k-1} \equiv e^{c_{k}-c_{1}} & =\left|\left\langle\mathcal{X}_{1}\right\rangle\right|^{2}\left|\left\langle\mathcal{X}_{k}\right\rangle\right|^{-2 / k} \\
\frac{d \mathcal{I}}{\mathcal{I}}=-\frac{d\left\langle\mathcal{X}_{1}\right\rangle}{(1 / 2)\left\langle\mathcal{X}_{1}\right\rangle} \quad \longrightarrow \quad \ln |\mathcal{I}|+c_{\mathcal{I}} & =-2 \ln \left|\left\langle\mathcal{X}_{1}\right\rangle\right|+c_{1} \\
\ln \left[e^{c_{\mathcal{I}}}|\mathcal{I}|\right] & =\ln \left[e^{c_{1}}\left|\left\langle\mathcal{X}_{1}\right\rangle\right|^{-2}\right] \\
e^{c_{\mathcal{I}}}|\mathcal{I}| & =e^{c_{1}}\left|\left\langle\mathcal{X}_{1}\right\rangle\right|^{-2} \\
& \downarrow \\
b_{M} \equiv e^{c_{1}-c_{\mathcal{I}}} & =\left|\left\langle\mathcal{X}_{1}\right\rangle\right|^{2}|\mathcal{I}| .
\end{aligned}
$$

We have now constructed an integral basis for the characteristic system (57)

$$
b_{1}=u_{1}\left(\left\langle\mathcal{X}_{1}\right\rangle, \ldots,\left\langle\mathcal{X}_{M}\right\rangle, \mathcal{I}\right), \ldots, b_{M}=u_{M}\left(\left\langle\mathcal{X}_{1}\right\rangle, \ldots,\left\langle\mathcal{X}_{M}\right\rangle, \mathcal{I}\right)
$$

and the general solution of equation (201) defined as

$$
\Phi\left(u_{1}, u_{2}, \ldots, u_{M}\right)=0
$$

is given by

$$
\Phi\left(\left|\left\langle\mathcal{X}_{1}\right\rangle\right|^{2}\left|\left\langle\mathcal{X}_{2}\right\rangle\right|^{-1}, \cdots,\left|\left\langle\mathcal{X}_{1}\right\rangle\right|^{2}\left|\left\langle\mathcal{X}_{k}\right\rangle\right|^{-2 / k}, \cdots,\left|\left\langle\mathcal{X}_{1}\right\rangle\right|^{2}\left|\left\langle\mathcal{X}_{M}\right\rangle\right|^{-2 / M},\left|\left\langle\mathcal{X}_{1}\right\rangle\right|^{2}|\mathcal{I}|\right)=0
$$

where $\Phi$ is an arbitrary function of the $M$ variables. Solving this equation for $\mathcal{I}$ yields a solution of the explicit form

$$
\mathcal{I}=\left|\left\langle\mathcal{X}_{1}\right\rangle\right|^{-2} \Psi\left(\left|\left\langle\mathcal{X}_{1}\right\rangle\right|^{2}\left|\left\langle\mathcal{X}_{2}\right\rangle\right|^{-1}, \cdots,\left|\left\langle\mathcal{X}_{1}\right\rangle\right|^{2}\left|\left\langle\mathcal{X}_{k}\right\rangle\right|^{-2 / k}, \cdots,\left|\left\langle\mathcal{X}_{1}\right\rangle\right|^{2}\left|\left\langle\mathcal{X}_{M}\right\rangle\right|^{-2 / M}\right),(62
$$

where $\Psi$ is an arbitrary function of $(M-1)$ variables.

\section{Cauchy problem and the existence and uniqueness of the solution to our PDE}

One of the fundamental aspects so as to have a useful PDE for modeling physical systems revolves around the existence and uniqueness of the solutions to the Cauchy problem. Here we show that such requirements are satisfied by our pertinent solutions. We start by casting (20) in the normal form

$$
\frac{\partial \mathcal{I}}{\partial\left\langle\mathcal{X}_{1}\right\rangle}=F\left(\left\langle\mathcal{X}_{1}\right\rangle, \cdots,\left\langle\mathcal{X}_{M}\right\rangle, \mathcal{I}, \frac{\partial \mathcal{I}}{\partial\left\langle\mathcal{X}_{2}\right\rangle}, \cdots, \frac{\partial \mathcal{I}}{\partial\left\langle\mathcal{X}_{M}\right\rangle}\right)
$$


where

$$
F_{\mathcal{I}}=F\left(\left\langle\mathcal{X}_{1}\right\rangle, \cdots,\left\langle\mathcal{X}_{M}\right\rangle, \mathcal{I}, \frac{\partial \mathcal{I}}{\partial\left\langle\mathcal{X}_{2}\right\rangle}, \cdots, \frac{\partial \mathcal{I}}{\partial\left\langle\mathcal{X}_{M}\right\rangle}\right)=-\frac{2}{\left\langle\mathcal{X}_{1}\right\rangle}\left[\mathcal{I}+\sum_{k=2}^{M} \frac{k}{2}\left\langle\mathcal{X}_{k}\right\rangle \frac{\partial \mathcal{I}}{\partial\left\langle\mathcal{X}_{k}\right\rangle}\right]
$$

and we see that $F$ is a real function of class $C^{2}$ in a neighborhood of

$$
\left\langle\mathcal{X}_{1}\right\rangle=a,\left\langle\mathcal{X}_{k}\right\rangle=\xi_{k-1}, \mathcal{I}\left(\xi_{1}, \ldots, \xi_{M-1}\right)=c,\left.\frac{\partial \mathcal{I}}{\partial\left\langle\mathcal{X}_{k}\right\rangle}\right|_{\xi_{1}, \ldots, \xi_{M-1}}=d_{k-1}, \quad k=2, \cdots, M
$$

Then, if $\psi\left(\left\langle\mathcal{X}_{2}\right\rangle, \ldots,\left\langle\mathcal{X}_{M}\right\rangle\right)$ is also a function of class $C^{2}$ such that

$$
\psi\left(\xi_{1}, \ldots, \xi_{M-1}\right)=c,\left.\quad \frac{\partial \psi}{\partial\left\langle\mathcal{X}_{k}\right\rangle}\right|_{\xi_{1}, \ldots, \xi_{M-1}}=d_{k-1}, \quad k=2, \ldots, M .
$$

exists a solution $\mathcal{I}$ of (63) in a neighborhood of $\left\langle\mathcal{X}_{1}\right\rangle=a$ and $\left\langle\mathcal{X}_{k}\right\rangle=\xi_{k-1}$ that satisfies

$$
\mathcal{I}\left(a,\left\langle\mathcal{X}_{2}\right\rangle, \cdots,\left\langle\mathcal{X}_{M}\right\rangle\right)=\psi\left(\left\langle\mathcal{X}_{2}\right\rangle, \cdots,\left\langle\mathcal{X}_{M}\right\rangle\right)
$$

and is of class $C^{2}$.

Regarding Cauchy-uniqueness, it is known that if $F$ satisfies the Lipschitz condition [35],

$$
\begin{gathered}
\left|F_{\mathcal{I}^{\prime}}-F_{\mathcal{I}}\right| \leq K_{1} \sum_{k=2}^{M}\left|\frac{\partial \mathcal{I}^{\prime}}{\partial\left\langle\mathcal{X}_{k}\right\rangle}-\frac{\partial \mathcal{I}}{\partial\left\langle\mathcal{X}_{k}\right\rangle}\right|+K_{2}\left|\mathcal{I}^{\prime}-\mathcal{I}\right| \quad K_{1}, K_{2}=\text { const. } \\
F_{\mathcal{I}}{ }^{\prime}=F\left(\left\langle\mathcal{X}_{1}\right\rangle, \cdots,\left\langle\mathcal{X}_{M}\right\rangle, \mathcal{I}^{\prime}, \frac{\partial \mathcal{I}^{\prime}}{\partial\left\langle\mathcal{X}_{2}\right\rangle}, \cdots, \frac{\partial \mathcal{I}^{\prime}}{\partial\left\langle\mathcal{X}_{M}\right\rangle}\right)
\end{gathered}
$$

then, the solution of the initial value problem for $(\underline{63})$ is unique. Note that in our case the above condition is verified always since the Legendre structure the theory guarantee that

$$
F_{\mathcal{I}}=\frac{\partial \mathcal{I}}{\partial\left\langle\mathcal{X}_{1}\right\rangle} \propto \frac{\partial I}{\partial\langle x\rangle}=\lambda_{1}<\infty
$$

\section{CONCLUSIONS}

It was known for some time [6] that, for one-dimensional scenarios, a minimal Fisher's information measure $I$ is associated to a Schrödinger's equation in which the role of the potential is played by a weighted sum of the a priori known expectation values of $M$ quantities $\left\langle x^{k}\right\rangle$. The weights are the corresponding Lagrange multipliers $\lambda_{k}$. Legendre reciprocity relations linking $I$, the $\lambda_{k}$ and the $\left\langle x^{k}\right\rangle$ apply. 
We have here demonstrated that insertion of virial theorem-tenets into this Legendre structure leads to a differential equation for $I$. The equation is analytically solvable and its solution provides us with explicit new expressions for $I$ in terms of the input-information contained in the $M$ expectation values $\left\langle x^{k}\right\rangle$. In other words, we can directly codify the information provided by such set of expectation values in an $I$-form without previous appeal to a Schrödinger equation. Additionally, this (partial) differential equation (PDE) may be viewed as a new constraint that the solutions $\psi_{n}$ of Schrödinger's equation (SE) must satisfy. In this regard, our PDE may lead to useful criteria for checking approximate treatments of SE. If $\phi_{n}$ is an approximate SE-solution, the fact that the functional $I\left[\phi_{n}\right]$ verifies the PDE would constitute an indicator of the quality of the approximate solution $\phi_{n}$.

An application to simple examples has illustrated these considerations. Of course, as is the case in the MaxEnt environment, the usefulness of (26) depends on how adequate is our input information for describing the situation at hand. Maximal entropy or minimum FIM are just the best ways to exploit that knowledge.

\section{Appendix: Translation transform of FIM}

The potential function

$$
U(x)=-\frac{1}{8} \sum_{k=1}^{M} \lambda_{k} x^{k} .
$$

can be Taylor-expanded about $x=\xi$

$$
U(x)=\sum_{k=0}^{M} \frac{U^{(k)}(\xi)}{k !}(x-\xi)^{k}
$$

The translational transform $u=x-\xi$ leads to

$$
\bar{U}(u)=U(u+\xi)=\sum_{k=0}^{M} \frac{U^{(k)}(\xi)}{k !} u^{k},
$$

which can be recast as

$$
\bar{U}(u)=-\frac{1}{8} \sum_{k=0}^{M} \lambda_{k}^{*} u^{k}
$$


with

$$
\lambda_{k}^{*} \equiv-8 \frac{U^{(k)}(\xi)}{k !}=-\frac{8}{k !} \sum_{j=1}^{M} j(j-1)(j-2) \cdots(j-k+1) \lambda_{j} \xi^{j-k} .
$$

The FIM-translational transform $u=x-\xi$ is obtained from (8) in the fashion $\left(\langle\rangle^{\prime}\right.$ indicates that the moment is calculated for translation-transform eigenfunctions)

$$
I=-4 \int \psi \frac{\partial^{2}}{\partial x^{2}} \psi d x=-4 \int \bar{\psi} \frac{\partial^{2}}{\partial u^{2}} \bar{\psi} d u=-4\left\langle\frac{\partial^{2}}{\partial u^{2}}\right\rangle^{\prime},
$$

where $\bar{\psi}=\bar{\psi}(u)$ is the translation transform of $\psi(x)$. Now, using the translation transform of (6) one easily finds

$$
I=\int \bar{\psi}_{n}\left(\alpha+\sum_{k=0}^{M} \lambda_{k}^{*} u^{k}\right) \bar{\psi}_{n} d u
$$

and one realizes that

$$
I=\alpha+\sum_{k=0}^{M} \lambda_{k}^{*}\left\langle u^{k}\right\rangle^{\prime}=\bar{\alpha}+\sum_{k=1}^{M} \lambda_{k}^{*}\left\langle u^{k}\right\rangle^{\prime}
$$

where

$$
\bar{\alpha}=\alpha+\lambda_{0}^{*}=\alpha-8 U(\xi)
$$

Also, the virial theorem (13) leads to

$$
I=4\left\langle\frac{\partial^{2}}{\partial u^{2}}\right\rangle^{\prime}=-4\left\langle u \frac{\partial}{\partial u} \bar{U}(u)\right\rangle^{\prime}=-\sum_{k=1}^{M} \frac{k}{2} \lambda_{k}^{*}\left\langle u^{k}\right\rangle^{\prime}
$$

The translation-transformed moments $\left\langle u^{k}\right\rangle^{\prime}$ are related to the original moments as

$$
\left\langle u^{k}\right\rangle^{\prime}=\int u^{k} \bar{\psi}^{2}(u) d u=\int u^{k} \psi^{2}(u+\xi) d u=\int(x-\xi)^{k} \psi^{2}(x) d x=\left\langle(x-\xi)^{k}\right\rangle
$$

By recourse to the Newton-binomial we write

$$
\int(x-\xi)^{k} \psi^{2}(x) d x=\sum_{j=1}^{k}(-1)^{j}\left(\begin{array}{c}
k \\
j
\end{array}\right) \xi^{j} \int x^{k-j} \psi^{2}(x) d x,
$$


and then we finally have

$$
\left\langle u^{k}\right\rangle^{\prime}=\left\langle(x-\xi)^{k}\right\rangle=\sum_{j=1}^{k}(-1)^{j}\left(\begin{array}{c}
k \\
j
\end{array}\right) \xi^{j}\left\langle x^{k-j}\right\rangle .
$$

Acknowledgment: Partial support from the programs FQM-2445 and FQM-207 of the Junta de Andalucia-Spain and from CONICET (Argentine Agency) is acknowledged.

[1] R. Carroll, On the emergence theme in physics (World Scientific, Singapore, 2010).

[2] P. Garbazcewkski, J. Stat. Phys. 123 (2006) 315.

[3] B. R. Frieden, Phys. Rev. A 41 (1990) 4265.

[4] B. R. Frieden, Physics from Fisher information (Cambridge, University Press; Cambridge, 1998); Science from Fisher information (Cambridge, University Press; Cambridge, 2004).

[5] B. R. Frieden, B. H. Soffer, Phys. Rev. E 521995 (1995) 2274.

[6] B. R. Frieden, A. Plastino, A. R. Plastino, B. H. Soffer, Phys. Rev. E 601999 (1999) 48.

[7] S.P. Flego, A. Plastino, A.R. Plastino, Physica A 390 (2011) 2276.

[8] S. P. Flego, B. R. Frieden, A. Plastino, A. R. Plastino, B. H. Soffer, Phys. Rev. E 68 (2003) 016105.

[9] M. Reginatto, Phys. Rev. E 58 (1998) 1775.

[10] M. Hall, Phys. Rev. A 62 (2000) 012107.

[11] V. Kapsa, L. Skala and J. Chen, Physica E 42 (2010) 293.

[12] B.R. Frieden and B.H. Soffer, Physica A 388 (2009) 1315.

[13] M. R. Ubriaco. Phys. Lett. A 373 (2009) 4017.

[14] S. Lopez-Rosa, J.C. Angulo, J.S. Dehesa and R.J. Yanez, Physica A 387 (2008) 2243.

[15] S. P. Flego, F. Olivares, A. Plastino, M. Casas, Entropy 13 (2011) 184.

[16] K.D. Sen, J. Antolin and J.C. Angulo, Phys. Rev. A 76 (2007) 032502.

[17] A. Nagy, Chem. Phys. Lett. 449 (2007) 212.

[18] A. Nagy, Chem. Phys. Lett. 425 (2006) 154.

[19] A. Hernando, C. Vesperinas, A. Plastino, Physica A 389 (2010) 490.

[20] A. Hernando, C. Vesperinas, A. Plastino, Phys. Lett. A 374 (2009) 18.

[21] F. Pennini, G. L. Ferri, A. Plastino, ENTROPY 11 (2009) 972. 
[22] F. Olivares, F. Pennini, A. Plastino, Physica A 389 (2010) 2218.

[23] F. Pennini, A. Plastino, B. H. Soffer, C. Vignat, Phys. Lett. A (2009) 373 (2009) 817.

[24] F. Pennini, A. Plastino, Phys. Rev. E 71 (2005) 047102.

[25] A. Katz, Principles of Statistical Mechanics, The Information Theory Approach (Freeman, San Francisco, 1967).

[26] R. P. Feynman, Phys. Rev. 56 (1939) 340.

[27] E. A. Desloge Thermal Physics (Holt, Rinehart and Winston, New York, 1968).

[28] For any quantum system in stationary state, with a Hamiltonian does not involve time explicitly, $H=-\frac{\hbar^{2}}{2 m} \vec{\nabla}^{2}+U(\vec{x})$, the virial theorem states that [29] $\left\langle-\frac{\hbar^{2}}{m} \vec{\nabla}^{2}\right\rangle=\langle\vec{x} . \vec{\nabla} U(\vec{x})\rangle$, where the expectation value is taken for stationary states of the Hamiltonian.

[29] W. Geiner and B. Müller, Quantum mechanics. An Introduction(Springer, Berlin, 1988).

[30] S. P. Flego, A. Plastino, A. R. Plastino, Physica A 390 (2011) 2276.

[31] J. M. Cassesl, Basic quantum mechanics (McGraw Hill, New York, 1970).

[32] R. Courant, D. Hilbert, Methods of Mathematical Physics, Vol II (Wiley (Interscience), New York, 1962); L.C. Evans, Partial Differential Equations (American Mathematical Society, Providence, 1998); A. D. Polyanin, V. F. Zaitsev, A. Moussiaux, Handbook of First Order Partial Differential Equations (Taylor \& Francis, London, 2002); A. D. Polyanin, Handbook of Linear Partial Differential Equations for Engineers and Scientists (Chapman \& Hall/CRC Press, Boca Raton, 2002).

[33] E. Kambe, Differentialgleichungen: Lösungsmethodem und Lösungen,II, Partielle Differentialgleichungen Erster Ordnung für eine gesuchte Funktion, (Akad, Verlagsgesellschaft Geest \& Portig, Leipzig, 1965).

[34] H. Rhee, R. Aris, N. R. Amundson, First Order Partial Differential Equations, Vol.I (Prentice Hall, Englewood Cliffs, New Jersey, 1986).

[35] Kiyosi Ito, Encyclopedic Dictionary of Mathematics-EDM 2-, 2 Ed. (MIT Press, Boston, 1996). 